\title{
ErbB4 Null Mice Display Altered Mesocorticolimbic and Nigrostriatal Dopamine Levels as well as Deficits in Cognitive and Motivational Behaviors
}

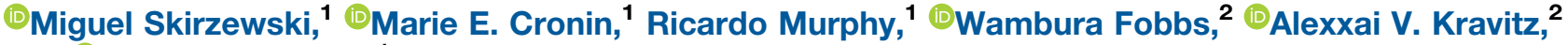 \\ and Andres Buonanno ${ }^{1}$
}

https://doi.org/10.1523/ENEURO.0395-19.2020

${ }^{1}$ Section on Molecular Neurobiology, Eunice Kennedy Shriver National Institute of Child Health and Human Development, Bethesda, MD 20892 and ${ }^{2}$ Eating and Addiction Section, Diabetes, Endocrinology, and Obesity Branch, National Institute of Diabetes and Digestive and Kidney Diseases, Bethesda, MD 20892

\begin{abstract}
Natural genetic variants of Neuregulin1 (NRG1) and its cognate receptor ErbB4 are associated with a risk for schizophrenia. Whereas most studies on NRG1-ErbB4 signaling have focused on GABAergic interneurons, ErbB4 is also expressed by midbrain dopaminergic neurons where it modulates extracellular dopamine (DA) levels. Here, we report that extracellular steady-state levels of DA are reduced in the medial prefrontal cortex (mPFC; $-65 \%)$, hippocampus (-53\%) and nucleus accumbens (NAc; $-35 \%)$, but are elevated in the dorsal striatum (+25\%) of ErbB4 knock-out mice (ErbB4 KOs) relative to wild-type controls. This pattern of DA imbalance recapitulates the reported prefrontal cortical reduction and striatal increase of DA levels in schizophrenia patients. Next, we report on a battery of behavioral tasks used to evaluate locomotor, cognitive and motivational behaviors in ErbB4 KOs relative to controls. We found that ErbB4 KOs are hyperactive in a novel open field but not in their familiar home cage, are more sensitive to amphetamine, perform poorly in the T-maze and novel object recognition (NOR) tasks, exhibit reduced spatial learning and memory on the Barnes maze, and perform markedly worse in conditioned place preference (CPP) tasks when associating cued-reward palatable food with location. However, we found that the poor performance of ErbB4 KOs in CPP are likely due to deficits in spatial memory, instead of reward seeking, as ErbB4 KOs are more motivated to work for palatable food rewards. Our findings indicate that ErbB4 signaling affects tonic DA levels and modulates a wide array of behavioral deficits relevant to psychiatric disorders, including schizophrenia.
\end{abstract}

Key words: cognition; dopamine; ErbB4; locomotion; motivation

\section{Significance Statement}

Neuregulins (NRGs) and their major neuronal receptor in the brain, ErbB4, have been genetically associated with schizophrenia. In rodents, ErbB4 signaling has been shown to acutely regulate intrinsic interneuron excitability, synaptic plasticity, neuronal network activity and extracellular dopamine (DA) levels. Here we report that ErbB4 null mutant mice exhibit an imbalance of extracellular DA levels relative to controls, with increased levels in the dorsal striatum but reduced levels in the prefrontal cortex (PFC), hippocampus, and nucleus accumbens (NAc); a similar striatal and cortical DA imbalance has been reported in subjects with schizophrenia. Additionally, we show that ErbB4 null mice exhibit deficits in cognitive-related tasks, locomotor activity and motivation in a battery of behavioral assays previously reported to be associated with DA levels. 


\section{Introduction}

Neuregulins (NRGs) are a family of neurodevelopmental factors comprised of four genes (NRG1-NRG4) encoding proteins harboring a conserved epithelial growth factorlike (EGF-L) domain necessary for binding and activation of ErbB receptor tyrosine kinases (Buonanno, 2010; Mei and Nave, 2014). The major neuronal NRG receptor in the brain is ErbB4, where it regulates GABAergic neuronal migration, excitatory glutamatergic, cholinergic and inhibitory synapses onto GABAergic neurons (Chang and Fischbach, 2006; Zhong et al., 2008; Mitchell et al., 2013; Vullhorst et al., 2015), intrinsic interneuron excitability ( $\mathrm{Li}$ et al., 2011; Janssen et al., 2012), synaptic plasticity (Kwon et al., 2005; Chen et al., 2010), neuronal network activity (i.e., $\gamma$ oscillations; Fisahn et al., 2009; Andersson et al., 2012; Kawata et al., 2017), closure of the visual critical period (Sun et al., 2016; Grieco et al., 2019), and extracellular dopamine (DA) levels (Kwon et al., 2008; Namba et al., 2016; Skirzewski et al., 2018).

Importantly, numerous independent studies have identified a genetic association of natural variants of NRG1, NRG3, and ErbB4 with schizophrenia (Law et al., 2007; Kao et al., 2010; Greenwood et al., 2012; Joshi et al., 2014; Mostaid et al., 2016), as well as with altered electrophysiological properties of inducible pluripotential stem cells isolated from schizophrenia patients (Brennand et al., 2011). Postmortem analyses of the dorsal lateral prefrontal cortex (PFC) of patients indicate an alteration in the relative ratio of NRG1 and ErbB4 splice variants relative to controls (Law et al., 2006; Bertram et al., 2007; Chong et al., 2008). In this regard it is important that mice with targeted mutations in either nrg1, nrg2, nrg3, or erbb4 exhibit a number of behavioral deficits that are relevant to traits affected in schizophrenia (Chen et al., 2010; Wen et al., 2010; Shamir et al., 2012; Lu et al., 2014; Hayes et al., 2016; Yan et al., 2018), and in two of the studies that

Received September 29, 2019; accepted February 27, 2020; First published April 30, 2020.

The authors declare no competing financial interests.

Author contributions: M.S., A.V.K., and A.B. designed research; M.S., M.E.C., R.M., and W.F. performed research; M.S. analyzed data; M.S. and A.B. wrote the paper.

This work was supported by the Intramural Research Programs of the Eunice Kennedy Shriver National Institute of Child Health and Human Development Grant ZIA-HD000711 (to M.S., M.E.C., R.M., and A.B.), the National Institute of Diabetes and Digestive and Kidney Diseases (W.F. and A.V.K.), and the National Alliance for Research on Schizophrenia and Depression Young Investigator Grant 27461 (to A.V.K.).

M. Skirzewski's present address: Rodent Cognition Core, Schulich Robarts Research Institute, University of Western Ontario, London, Ontario N6G 5B7, Canada.

A. Kravitz's present address: Departments of Psychiatry, Anesthesiology, and Neuroscience, Washington University, St. Louis, MO 63110.

Acknowledgments: We thank Daniel Abebe for help with animal care and Oren Princz-Lebel for proofreading this manuscript.

Correspondence should be addressed to Andres Buonanno at buonanno@ mail.nih.gov.

https://doi.org/10.1523/ENEURO.0395-19.2020

Copyright (C) 2020 Skirzewski et al.

This is an open-access article distributed under the terms of the Creative Commons Attribution 4.0 International license, which permits unrestricted use, distribution and reproduction in any medium provided that the original work is properly attributed. tested the effects of antipsychotics in mutant mice, the behavioral deficits observed were improved (Tan et al., 2018; Yan et al., 2018).

In the cortex and hippocampus, cellular ErbB4 expression is confined to GABAergic interneurons (Vullhorst et al., 2009; Fazzari et al., 2010; Neddens and Buonanno, 2010; Neddens et al., 2011; Del Pino et al., 2013; Bean et al., 2014). ErbB4 levels are especially high in parvalbumin (PV)-positive interneurons, where receptor expression regulates $\gamma$ oscillations (Fisahn et al., 2009; Chen et al., 2010; Fazzari et al., 2010; Wen et al., 2010; Shamir et al., 2012; Sun et al., 2016; Tan et al., 2018), a type of neuronal network activity important for working memory and other cognitive processes (Uhlhaas and Singer, 2010; Lewis et al., 2011; Miller et al., 2018). In contrast to the extensively studied function of ErbB4 in GABAergic interneurons, much less is known about the contribution of ErbB4 in mesencephalic DA neurons. Acute local administration of NRG1 (1 nM) by reverse microdialysis rapidly increases extracellular DA levels in the dorsal hippocampus, medial PFC (mPFC), and dorsal striatum within minutes (Kwon et al., 2008; Skirzewski et al., 2018). The increases of extracellular DA levels by NRG result from the activation of ErbB4 and downstream inhibition of the DA transporter (DAT), which is expressed on axonal processes (Skirzewski et al., 2018). Moreover, chronic disruption of NRG or ErbB4 signaling in knock-out (KO) mice alter tonic DA levels in the mPFC, hippocampus, and striatum (Kato et al., 2010, 2011; Mizuno et al., 2013; Golani et al., 2014; Tadmor et al., 2017, 2018; Skirzewski et al., 2018; Yan et al., 2018).

Despite the emerging literature associating NRG-ErbB signaling with DA function, how mutation of ErbB4 affects the nigrostriatal, mesocortical, and mesolimbic DA systems is presently unknown. Here we report that tonic extracellular levels of DA and its metabolites 3,4-dihydroxyphenylacetic acid (DOPAC) and homovanilic acid (HVA) in ErbB4 KOs are inversely disrupted between the dorsal striatum versus the mPFC, dorsal hippocampus, and nucleus accumbens (NAc) relative to controls. We also show that ErbB4 KO mice reproduce several behavioral deficits associated with altered striatal, hippocampal and/or cortical function that are relevant to psychiatric disorders, including schizophrenia.

\section{Materials and Methods}

\section{Animals}

We used a line of null ErbB4 KO mice originally developed by Tidcombe et al. (2003), which circumvents embryonic lethality by selective transgenic expression of ErbB4 in the heart driven by the myosin heavy chain promoter. Adult male ErbB4 KO and wild-type C57BL/6J controls (hereafter Ctrl) mice (two to five months old) were used. Mice were in a "clean" C57BL/6J background ( $>20$ generations backcrossed to C57BL/6J) and housed in a conventional $12 / 12 \mathrm{~h}$ light/dark schedule with access to food and water ad libitum at the National Institutes of Health (NIH). Animal procedures were reviewed and approved by the NIH Animal Care and Use Committee. 


\section{Reagents}

Regular chow food (5001 Rodent Diet, $3.0 \mathrm{kcal} / \mathrm{g}$ with $29 \%$ energy derived from protein, $13 \%$ from fat, and $56 \%$ from carbohydrate) was obtained from LabDiet. Dustless Precision Pellets $(14 \mathrm{mg}, 3.6 \mathrm{kcal} / \mathrm{g})$ were from BioServ. Amphetamine hydrobromide, neurochemical standards and HPLC reagents were from Sigma-Aldrich.

\section{Microdialysis}

Extracellular DA, DOPAC, and HVA levels in the dorsal striatum, NAc, dorsal hippocampus, and mPFC were measured using in vivo microdialysis in freely moving mice, as previously reported (Skirzewski et al., 2018). Guide cannulas (stainless-steel $7 \mathrm{~mm}$ long, 21 gauge) were unilaterally implanted in the striatum (AP: $+0.5 \mathrm{~mm}$, L: $1.8 \mathrm{~mm}, \mathrm{~V}: 1.1 \mathrm{~mm}$ ), NAc (AP: $+1.4 \mathrm{~mm}$, L: $0.5 \mathrm{~mm}$, V: $2.2 \mathrm{~mm}$ ), or mPFC (AP: $+2.0 \mathrm{~mm}$, L: $0.3 \mathrm{~mm}, \mathrm{~V}: 0.3 \mathrm{~mm}$ ), and they were bilaterally implanted in the dorsal hippocampus (AP: $-2.5 \mathrm{~mm}, \mathrm{~L}: 2.5 \mathrm{~mm}, \mathrm{~V}: 0.0 \mathrm{~mm}$ ); the AP, L, and $V$ values correspond to measurements relative to bregma, midsagittal sinus, and brain surface (Paxinos and Franklin, 2001). After surgery performed with $2 \%$ isoflurane/oxygen anesthesia, mice were allowed to recuperate for a week before starting microdialysis. Microdialysis probes were prepared in the laboratory, as described (Hernandez et al., 1986), and consisted of an 8-mm-long, 26-gauge, stainless steel tube plus a 2-mm-long (mPFC, striatum) or 1-mm-long (NAc, hippocampus) cellulose hollow fiber tip (18 kDA MWCO, SpectrumLabs Inc) that protruded from the guide cannula. Modified ACS fluid (136 $\mathrm{mm} \mathrm{NaCl}$, $3.7 \mathrm{~mm} \mathrm{KCl}, 2.2 \mathrm{~mm} \mathrm{CaCl}_{2}, 1 \mathrm{~mm} \mathrm{MgCl}_{2}$, and 10 $\mathrm{mm} \mathrm{NaHCO}_{3}$ at $\mathrm{pH}$ 7.4) was perfused through the probes at a flow rate of $1 \mu \mathrm{l} / \mathrm{min}$.

Five samples were collected consecutively for $15 \mathrm{~min}$ from each mouse into tubes containing $5 \mu$ of $100 \mathrm{~mm}$ $\mathrm{HCl}+1 \mathrm{~mm}$ EDTA, which were immediately frozen in dryice following collection to prevent catecholamine oxidation. Samples from two genotype-matched mice were pooled to enable measurement of DA collected from the hippocampus and mPFC using electrochemistry (see HPLC-electrochemical detection). To estimate extracellular concentration of DA, DOPAC, and HVA, the percentage of recovery was obtained in vitro for each microdialysis probe. Mice used to measure DA levels were sacrificed following sample collection to confirm the anatomic placement of the probe. Brains were fixed by trans-cardiac perfusion using $30 \mathrm{ml} \mathrm{PBS,} \mathrm{pH} 7.4$, followed by $30-\mathrm{ml} 4 \%$ formaldehyde in PBS, sectioned $(50 \mu \mathrm{m}$ thick), and Nissl stained to verify probe placement (Fig. $1 B, D, F, H)$. Brains with misplaced microdialysis probes were excluded from data analysis.

\section{HPLC-electrochemical detection}

The extracellular content of DA, DOPAC, and HVA from dialyzed samples was analyzed by injecting the total sample volume into an isocratic HPLC system with electrochemical detection (Model LC-4C, BASi), essentially as published (Skirzewski et al., 2018; Yan et al., 2018). The order and elution time of the neurochemicals was as follows: DOPAC $\sim 7.0 \mathrm{~min}, \mathrm{DA} \sim 9.5 \mathrm{~min}$, and HVA $\sim 21.9 \mathrm{~min}$. DA, DOPAC, and HVA were measured by matching the area under the curve (AUC) for each sample versus the AUC for the curves for each standard; then the amount was corrected for the percent recovery for the dialysis probe corresponding to each molecule. Detection sensitivity limit was set to $1 \mathrm{nA}$ for DA, DOPAC, and HVA, estimated to correspond to 2.4, 1.3, and $2.7 \mathrm{pg}$, respectively.

\section{Behavioral testing battery}

We performed and compared a battery of behavioral tasks in ErbB4 KOs and Ctrl mice (three to five months old) using cohorts that varied in number (5-13 mice) depending on the test used. As previously suggested (Crawley, 2007), and to minimize/optimize the number of animals used in this study, we used eight independent cohorts of mice that were tested in no more than five consecutive behavioral tasks performed in the following order. Cohorts 1 and 2: home-cage locomotor activity, open field test, novel object recognition (NOR), T-maze, and Barnes maze. Cohort 3: auditory Pavlovian conditioning, progressive ratio (PR), and amphetamine challenge test. Cohort 4: two-bottle sucrose preference, auditory Pavlovian conditioning, PR, and conditioned place preference (CPP) to palatable food. Cohort 5: two-bottle sucrose preference, PR, and CPP to palatable food. Cohort 6: amphetamine challenge test. Cohort 7: energy balance/ food consumption. And cohort 8: body weight analysis. All behavioral tests were performed during the light period (12/12 h light/dark cycle).

\section{Locomotor activity in home cage}

ErbB4 KO and Ctrl mice were independently housed in a home cage $(19 \times 18 \times 40 \mathrm{~cm})$ with regular bedding, free access to food and water, and standard 12/12 h light/dark schedule for $96 \mathrm{~h}$. Horizontal locomotor activity was continuously recorded by placing the home cage within an infrared beam-equipped frame (Columbus Instruments).

\section{Open field test}

Novelty induced locomotor activity was tested in a $35 \times$ $35 \times 35 \mathrm{~cm}$ open field arena. Mice were independently placed in one corner of the arena and horizontal movements recorded during 30 min with Any-Maze Behavior Video Tracking Software V 4.72 (Stoelting Co). The open field chamber was cleaned between sessions with $70 \% \mathrm{v} /$ $v$ alcohol solution.

\section{Amphetamine challenge}

Systemic administration of amphetamine (intraperitoneal) in rodents induces an overall increase in locomotor activity (Bardo et al., 1990), and altered basal extracellular DA levels in the brain affects their amphetamine-induced locomotor response (Yan et al., 2018). ErbB4 KO and Ctrl mice were challenged with different doses of amphetamine $(0.0,0.5,1.5,2.5$, and $3.5 \mathrm{mg} / \mathrm{kg}$, i.p.). As described for the open field test (see above), horizontal locomotor activity was initially recorded in an open field arena $(35 \times$ $35 \times 35 \mathrm{~cm}$ ) for $60 \mathrm{~min}$ before administering a single injection of amphetamine or vehicle $(0.9 \% \mathrm{NaCl})$. Locomotor 
A

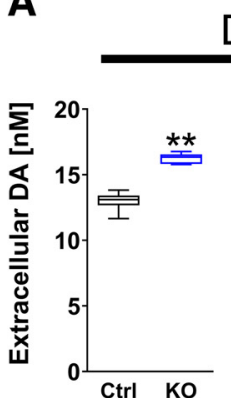

Dorsal Striatum
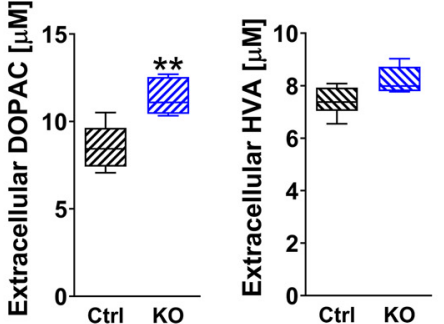

C

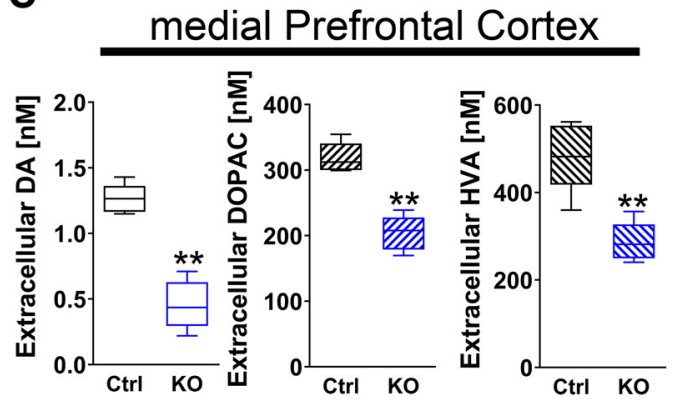

E

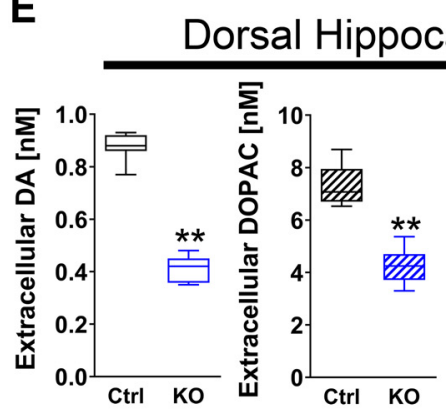

G

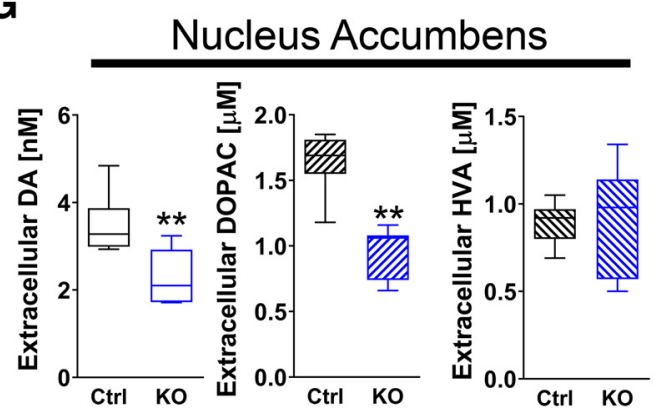

B

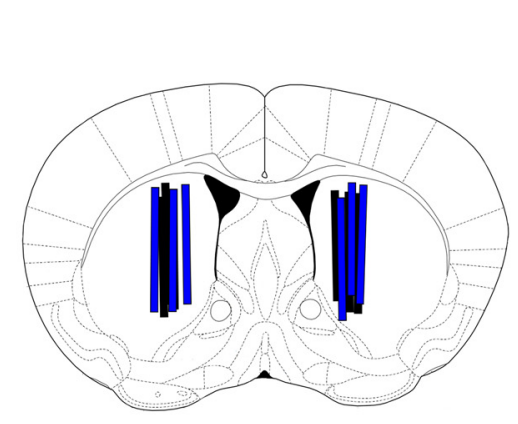

D 
objects placed at the distal portion of each arm. The remaining arm (start arm) did not have any object and was equipped with a guillotine door $(30 \mathrm{~cm}$ away from the center of apparatus) to prevent the mouse's free exploration. The sample trial started by raising the guillotine door at the start arm to allow the mouse to freely explore the apparatus and objects. During the choice trial, one of the objects was replaced by a new object of different shape and texture, but of similar size. The time mice spent in arms with novel versus familiar objects were video recorded using Any-Maze (Stoelting), and the exploration ratio (novel vs familiar) was assessed. The apparatus and objects were wiped down with $70 \% \mathrm{v} / \mathrm{v}$ ethanol between sessions.

\section{T-maze}

Spontaneous alternation behavior in mice reflects a sufficient level of reference and working memory to successfully explore for novel environments (Deacon and Rawlins, 2006). As previously described (Skirzewski et al., 2018), the test was assessed in an opaque plastic enclosed $T$ maze apparatus consisting of one start arm where the mouse started the trial and two choice arms equipped with Plexiglas doors at the entrance. Briefly, the task session consisted of a "sample" and a "choice" trial separated by 15-s ITI where the mouse remained in a clean home cage. The sample trial started after gently placing the mouse at the end of the start arm and allowing it to freely explore either the left or the right arm. The arm was defined as "chosen" when the mouse placed the four paws and tail inside an arm. Once inside the arm, the mouse was restricted to this compartment for $30 \mathrm{~s}$. The mouse was then gently retrieved out of the apparatus for ITI. Lastly, with all the doors opened, the choice trial was initiated by placing the mouse at the start arm and allowing it to freely explore the apparatus. Whether the mouse chose to explore the unvisited arm (alternation) or the previously visited arm (no alternation) was recorded. Spontaneous performance was scored in each mouse over three sessions that were separated by $2 \mathrm{~h}$ each.

\section{Barnes maze}

The Barnes maze was used to assess spatial learning memory, as previously described (Sunyer et al., 2007; Skirzewski et al., 2018). The apparatus consisted of an elevated white Plexiglas circular table $(92 \mathrm{~cm}$ in diameter) with 20 equally spaced holes $(5 \mathrm{~cm}$ in diameter, $7.5 \mathrm{~cm}$ between holes) along the perimeter. One of the holes (target) contained a hidden box underneath the table that allowed mice to hide from two mild stressors (85-db background noise/900 lux light) applied during the training trials (see below). The remaining 19 holes did not offer escape to the mild stressors and were sequentially numbered +1 to +9 , opposite to target, and -9 to -1 . Holes +1 and -1 corresponded to the adjacent holes to target. The task consisted in two phases: four training sessions (one per day, days 1-4) and probe trial (day 5). The training sessions consisted in 4 trials/session during four consecutive days (30-min ITI). Each trial was 3 min long or until the mouse escaped to the hidden box underneath the target, whichever came first. Time mice spent finding the target (latency time) and number of errors (nose pokes in other holes) for each trial were scored from day 1 to 4 . Probe trial (day 5) was recorded once per mouse during $90 \mathrm{~s}$, and the hidden box was removed from the apparatus. Latency time mouse entered for the first time to the correct quadrant (1/4 of the platform area including target and holes \pm 1 and \pm 2 ), total time spent in correct quadrant, and number of nose pokes to target and other holes were recorded using Any-Maze (Stoelting). The apparatus was wiped between trials with a $70 \% \mathrm{v} / \mathrm{v}$ alcohol solution.

\section{Two-bottle sucrose test}

The two-bottle sucrose test paradigm was used to determine anhedonia in ErbB4 KOs and Ctrl mice as previously described (Hutchison et al., 2018). Mice housed individually were habituated to drink tap water from two identical bottles during $24 \mathrm{~h}$. Then, mice were given a free choice to drink either $2 \%(\mathrm{v} / \mathrm{v})$ sucrose solution or tap water in two bottles and intake scored by weighting the bottles every $24 \mathrm{~h}$ for four consecutive days. To avoid side preference, the location of sucrose and water bottles were alternated every $24 \mathrm{~h}$. The sucrose preference ratio was calculated as the amount of sucrose solution consumed relative to the total amount of liquid consumed (sucrose solution intake/[sucrose solution intake + water intake]).

\section{CPP}

Using the five-test design (Hutchison et al., 2018), foodrestricted mice ( $85 \%$ body weight) were tested for their ability to form conditioned spatial associations to palatable food (14-mg dustless precision pellets, BioServ) in the CPP paradigm. The CPP apparatus consisted of two large compartments $(20 \times 20 \times 20 \mathrm{~cm})$ with different visual and tactile cues, which were separated by a smaller center compartment $(10 \times 10 \times 20 \mathrm{~cm})$. First, mice underwent in a pre-training session to identify their natural place preference by allowing them to freely explore the apparatus during $20 \mathrm{~min}$. A biased design at days 1, 3, 5, 7 , and 9 was used to administer a neutral stimulus (no food) in the naturally preferred side (PS) or 20 palatable food pellets sparsely distributed on the floor of the least PS (LPS). Mouse exploration was restricted to either PS or LPS during $15 \mathrm{~min}$ depending of stimulus (neutral or palatable food pellets). Probe sessions were held at days $2,4,6,8$, and 10 and consisted in a single trial to let mice to freely explore the entire apparatus during $20 \mathrm{~min}$ with no palatable food provided. Time spent at each compartment was recorded (LPS vs PS) using Any-Maze (Stoelting), and preference ratio estimated as the time spent in the LPS divided by the total time spent in PS and LPS.

\section{Auditory Pavlovian conditioning}

Food restricted ( $85 \%$ body weight) ErbB4 $\mathrm{KO}$ and Ctrl mice were used to assess deficits in associative learning behavior using a previously described paradigm (Berridge and Robinson, 2003). Experiments were performed in a sound-attenuated operant chamber equipped with a fan to provide white noise and ventilation during the experiment $(20 \times 20 \times 20 \mathrm{~cm}$, ENV-300; Med Associates). 
Briefly, mice were trained during $30 \mathrm{~min}$ to retrieve a palatable food reward (14-mg dustless precision pellets, BioServ) according to the following trial schedule: 2-s tone $(85 \mathrm{db}) \rightarrow 1$-s delay $\rightarrow 5$-s-long light on inside reward magazine $\rightarrow$ mouse retrieve reward within when reward magazine is illuminated $\rightarrow 15$-s ITI $\rightarrow$ repeat trial. If the mouse nose poked the reward magazine at any other time different from when the reward was active (light on), a time out was applied (5-s-long house light on) and the schedule resumed with 15-s ITI. Daily single sessions were performed for eight consecutive days and percentage of accuracy (rewards retrieved vs total trials) and premature responses (time out) scored.

\section{PR}

This task was used to test the motivation and willingness of ErbB4 KO and Ctrl mice to work for palatable food rewards (Berridge and Robinson, 2003). Mice were kept at $85 \%$ free-feeding body weight and conditioning was performed in sound-attenuated operant chambers (ENV300; Med Associates). Briefly, chambers were equipped with two illuminated nose-poke apertures on both sides of a reward magazine that dispensed palatable food pellets (14-mg dustless precision pellets, BioServ), and a fan that provided white noise and ventilation to the chamber during the session (Fig. 5A). One nose-poke aperture was set as active and the other as inactive, with the active location counterbalanced across mice. Nose poking in the active aperture resulted in the delivery of one palatable food pellet, while responses in the inactive aperture were recorded but had no consequence. Before conditioning, mice were habituated to retrieve rewards delivered at variable interval schedules (range 5-100 s) for $30 \mathrm{~min}$ and nose poke responses had no consequence. Then, mice were trained in a fixed ratio 1 (FR1) schedule of reinforcement where each nose poke in the active aperture resulted in the delivery of one palatable food pellet. Our criteria for stable performance was the collection of 30 food pellets in a 30-min-long session during three consecutive days. Once criteria were met, mice were progressively transferred to FR3 (three responses in active aperture - one reward) and FR5 (five responses in active aperture - one reward) schedules following the same criteria performance. Finally, one PR7 schedule session was assessed daily during five consecutive days to test for the mouse's motivation to work for rewards. PR7 session consisted in a progressive increment of the required nose pokes into the active aperture $(7,14,21,28,35$, etc.) per trial to obtain one palatable food pellet. The total number of palatable pellets collected (breaking point) and number of nose pokes in the active/inactive holes in a 2-h-long session or after 60 min of inactivity (whichever occurred first) were recorded.

Food intake, body weight, body composition, and energy balance

Three-month-old ErbB4 $\mathrm{KO}$ and Ctrl mice were single housed under standard conditions (12/12 h light/dark cycle, $21-22^{\circ} \mathrm{C}$ ) with ad libitum access to water and regular chow diet (LabDiet, 5001). Following one week of habituation, chow was presented in Rodent Cafes (OYC
Americas), and food intake and body weights were recorded daily for $10 \mathrm{~d}$. Food intake was measured by manually weighing the Rodent Cafes every $24 \mathrm{~h}$ and converting the weight consumed into calories using the metabolizable energy content. Body composition (fat and lean mass) was measured on the first and tenth day by $1 \mathrm{H}$ NMR spectroscopy (EchoMRI-100H; Echo Medical Systems LTD). Energy expenditure was calculated using the following equation: energy expenditure $=$ food intake $-(\Delta$ fat mass $+\Delta$ lean mass), as previously reported (Guo and Hall, 2009; Ravussin et al., 2013). Food consumption was presented as the total of energy intake (kcal) during $20 \mathrm{~min}$ and normalized by the mouse body weight before the session.

\section{Statistics}

Data were analyzed by using GraphPad Prism v8.2.1. Relative levels of DA and metabolites were estimated by comparing the AUC of each analyte with respect to the AUC of their corresponding standards. Additionally, extracellular concentration was corrected relative to the percentage of recovery for each microdialysis probe, which was calculated to be between $6-8 \%$ for a $1-\mathrm{mm}$-long tip and $10-15 \%$ for a $2-\mathrm{mm}$-long tip. All data were examined for normal distribution with D'Agostino and Pearson normality test. Unpaired two-tailed Student's $t$ test or twoway ANOVA for repeated measures and Sidak's multiple comparisons post hoc analysis was used with data showing normal distribution. If data set did not show normal distribution, two-tailed Mann-Whitney $U$ test was used. Data are represented as the mean \pm SEM, and significance was set at $p<0.05$.

\section{Results}

\section{ErbB4 KO mice show altered extracellular DA levels and metabolites across four different brain regions}

Although pharmacological or genetic manipulations of the NRG/ErbB4 signaling pathway have been reported to affect DA levels (Kato et al., 2010, 2011; Mizuno et al., 2013; Golani et al., 2014; Skirzewski et al., 2018; Yan et al., 2018), a systematic study of how genetic ablation of ErbB4 in adult mice alters tonic extracellular DA levels in distinct brain regions has not been undertaken. To address this point, we performed in vivo microdialysis coupled to HPLC/electrochemical neurochemical detection in freely moving ErbB4 KO and Ctrl mice to systematically measure extracellular DA, DOPAC, and HVA levels. Samples were collected from major dopaminergic brain regions comprised by the nigrostriatal (dorsal striatum), mesocortical (mPFC and dorsal hippocampus), and mesolimbic (NAc) systems. We found that extracellular DA levels (ErbB4 KO: $16.26 \pm 0.16 \mathrm{~nm}$ vs Ctrl: $13.00 \pm 0.29 \mathrm{~nm}$; $U=0, \quad p=0.0022$ ) and DOPAC levels (ErbB4 KO: $11.36 \pm 0.41 \mu \mathrm{M}$ vs Ctrl: $8.55 \pm 0.52 \mu \mathrm{M} ; U=2, p=0.0087$ ) were elevated in the dorsal striatum of ErbB4 KOs $(n=6)$ relative to controls $(n=6)$, whereas HVA levels (ErbB4 KO: $8.20 \pm 0.21 \mu \mathrm{M}$ vs Ctrl: $7.41 \pm 0.22 \mu \mathrm{M} ; p>0.05)$ were unchanged (Fig. 1A). The observation that DOPAC levels were higher, whereas HVA levels were similar between 
groups, is consistent with the notion that DA clearance in the striatum is mostly mediated by DAT uptake.

In contrast to the dorsal striatum, we found that extracellular levels of DA and its metabolites DOPAC and HVA were lower in both the mPFC (DA ErbB4 KO: $0.45 \pm 0.08 \mathrm{~nm}, n=6$ vs Ctrl: $1.27 \pm 0.05 \mathrm{~nm}, n=6, U=0$, $p=0.0022$; DOPAC ErbB4 KO: $205.10 \pm 10.91 \mathrm{~nm}$ vs Ctrl: $319.10 \pm 8.92 \mathrm{~nm}, U=0, p=0.0022$; HVA ErbB4 KO: $288.40 \pm 17.50$ nм vs Ctrl: $478.90 \pm 30.94$ nм; $U=0$, $p=0.0022$; Fig. $1 C$ ) and dorsal hippocampus (DA ErbB4 KO: $0.41 \pm 0.02 \mathrm{~nm}, n=6$ vs Ctrl $0.88 \pm 0.02 \mathrm{~nm}, n=7$, $U=0, p=0.0012$; DOPAC ErbB4 KO: $4.25 \pm 0.28$ nм vs Ctrl 7.38 $\pm 0.76 \mathrm{~nm}, \quad U=0, p=0.0012$; HVA ErbB4 KO: $18.66 \pm 0.87 \mathrm{~nm}$ vs Ctrl $32.31 \pm 1.13 \mathrm{~nm}, U=0$, $p=0.0012$; Fig. $1 E$ ) of ErbB4 KOs. Of note, we observed a similar imbalance of extracellular DA levels between striatal and extra-striatal structures in mice lacking the ErbB4 ligand NRG2 (Yan et al., 2018), suggesting that alterations in NRG-ErbB4 signaling in the whole brain differentially affect the nigrostriatal and mesocortical systems.

Because tonic DA levels are differentially altered in ErbB4 KO nigrostriatal (Fig. 1A) and mesocortical structures (Fig. $1 C, E$ ) relative to Ctrl mice, and the mesolimbic NAc comprises an anatomic structure that also receives inputs from the VTA, we were interested in investigating how mutation of ErbB4 affects tonic DA within the NAc. Interestingly, we found that extracellular DA levels (ErbB4 KO: $2.28 \pm 0.22 \mathrm{~nm}, n=7$ vs Ctrl: $3.51 \pm 0.25 \mathrm{~nm}, \quad n=7 ; \quad U=2, \quad p=0.0023)$ and DOPAC levels (ErbB4 KO: $0.94 \pm 0.07 \mu \mathrm{M}$ vs Ctrl $1.64 \pm 0.09 \mu \mathrm{M} ; U=0, p=0.0006)$, but not HVA (ErbB4 $\mathrm{KO} \quad 0.88 \pm 0.12 \mu \mathrm{M} ; \quad \mathrm{Ctrl} \quad 0.89 \pm 0.05 \mu \mathrm{M}, \quad p>0.05)$, were significantly reduced in ErbB4 KOs relative to Ctrl (Fig. 1G). The effects of ErbB4 ablation on NAc DA and DOPAC, but not on HVA, are consistent with relatively high expression levels of DAT in the NAc (ventral striatum) used to clear extracellular DA.

\section{ErbB4 KO mice are hyperactive and hypersensitive to amphetamine}

Changes in extracellular levels of neuromodulators in the striatum and neocortex have been associated with altered behaviors in rodents. For example, elevated tonic extracellular DA levels in the striatum have been associated with increased locomotor activity in rodents (Di Chiara and Imperato, 1988; Bardo et al., 1990). For this reason, we analyzed horizontal locomotor activity of ErbB4 $\mathrm{KO}$ mice relative to Ctrl in their home cage (i.e., habituated) and in the open field (i.e., novelty). Interestingly, we found that activity of ErbB4 KOs and Ctrl did not differ when recorded in their home cages during a 96-h period ( $n=8 /$ genotype, $p>0.05$; Fig. 2A), but in the open field ErbB4 KO mice traveled longer distances than Ctrl mice (ErbB4 KO: $81.6 \pm 3.0 \mathrm{~m}, n=9$; control: $48.7 \pm 2.7 \mathrm{~m}, n=9 ; F_{(2,24)}=$ $36.77 p<0.0001$; Fig. 2B). Moreover, ErbB4 KOs were hypersensitive to systemic amphetamine (intraperitoneal) relative to $\operatorname{Ctrl}\left(F_{(1,74)}=16.85, p=0.0001\right.$; Table 1 ; Fig. $2 C$ ), as their total horizontal locomotor activity was
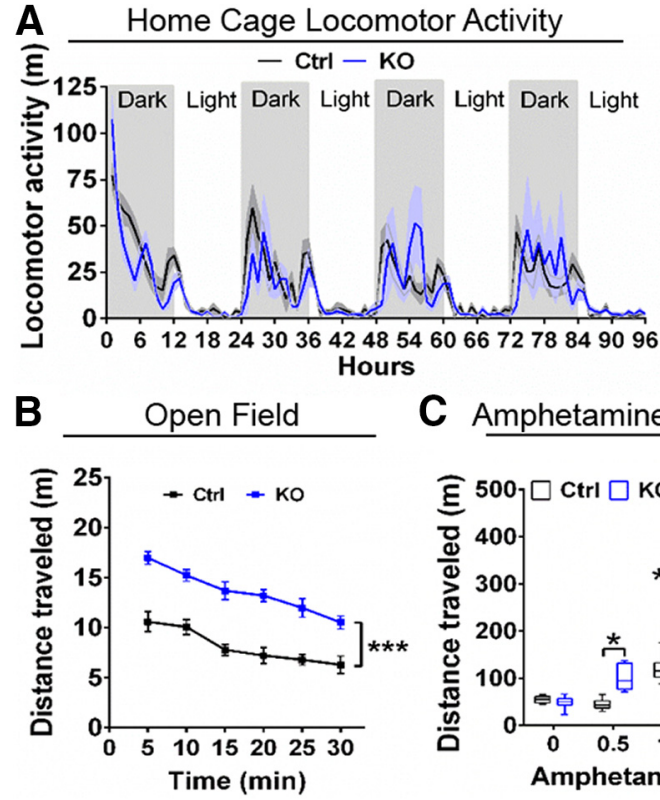

C Amphetamine Challenge

Figure 2. ErbB4 $\mathrm{KO}$ mice are hyperactive in novel environments and sensitive to systemic amphetamine. A, Horizontal locomotor activity of control (Ctrl, black) and ErbB4 KO (KO, blue) mice scored in their home cages for $96 \mathrm{~h}(n=8 /$ genotype, $p>0.05)$. $\boldsymbol{B}$, Locomotor activity of ErbB4 $\mathrm{KO}$ mice (KO, blue) is significantly elevated relative to controls (Ctrl, black) in the novelty open field test ( $n=9 /$ genotype, $\left.F_{(2,24)}=36.77, p<0.0001\right)$. $\boldsymbol{C}$, Locomotor activity of controls (Ctrl, black) and ErbB4 KO mice (KO, blue) following administration (intraperitoneal) of $0-3.5 \mathrm{mg} /$ $\mathrm{kg}$ amphetamine. ErbB4 $\mathrm{KO}$ mice $(0 \mathrm{mg} / \mathrm{kg}, n=9 ; 0.5-3.5 \mathrm{mg} /$ $\mathrm{kg}, n=8$ ) show locomotor hypersensitivity to systemic amphetamine administration at different doses as in contrast to Ctrl mice $(0 \mathrm{mg} / \mathrm{kg}, n=8 ; 0.5 \mathrm{mg} / \mathrm{kg}, n=10 ; 1.5$ and $2.5 \mathrm{mg} / \mathrm{kg}, n=8$; $\left.3.5 \mathrm{mg} / \mathrm{kg}, \quad n=9 ; \quad F_{(1,74)}=16.85, \quad p=0.0001\right) ; \quad * p<0.05$, $* * p<0.01, * * * p<0.001$.

higher at doses between $0.5-2.5 \mathrm{mg} / \mathrm{kg}$ and showed a reverse U-shaped response when the dose reached $3.5 \mathrm{mg} / \mathrm{kg}$ (intraperitoneal). The reversed U-shaped curve in response to amphetamine is consistent with previous work showing that that high doses of amphetamine resulted in stereotypical behaviors, rather than elevated horizontal locomotor activity (Segal, 1975). Moreover, these findings are also consistent with prior studies reporting ErbB4 KO mice are hyperactive when exposed to novel environments and to augmented basal levels of striatal DA in KOs (Shamir et al., 2012; Marchisella et al., 2018).

\section{Table 1: Total horizontal locomotor activity (meters) recorded for $60 \mathrm{~min}$ from ErbB4 KO and Ctrl mice that received systemic injections (intraperitoneally) of D-amphetamine ( $\mathrm{mg} / \mathrm{kg})$}

\begin{tabular}{llllll}
\hline $\mathrm{mg} / \mathrm{kg}$ & Ctrl & $n$ & ErbB4 KO & $n$ & $p$ \\
0.0 & $54.6 \pm 2.5$ & 8 & $49.3 \pm 4.1$ & 9 & n.s. \\
0.5 & $44.5 \pm 3.3$ & 10 & $105.8 \pm 9.5$ & 8 & $<0.05$ \\
1.5 & $121.2 \pm 9.4$ & 8 & $207.1 \pm 21.8$ & 8 & $<0.005$ \\
2.5 & $206.2 \pm 12.4$ & 8 & $326.6 \pm 22.8$ & 8 & $<0.001$ \\
3.5 & $279.1 \pm 17.1$ & 9 & $207.0 \pm 23.3$ & 8 & $<0.01$ \\
\hline
\end{tabular}

n.s., no significant differences. 

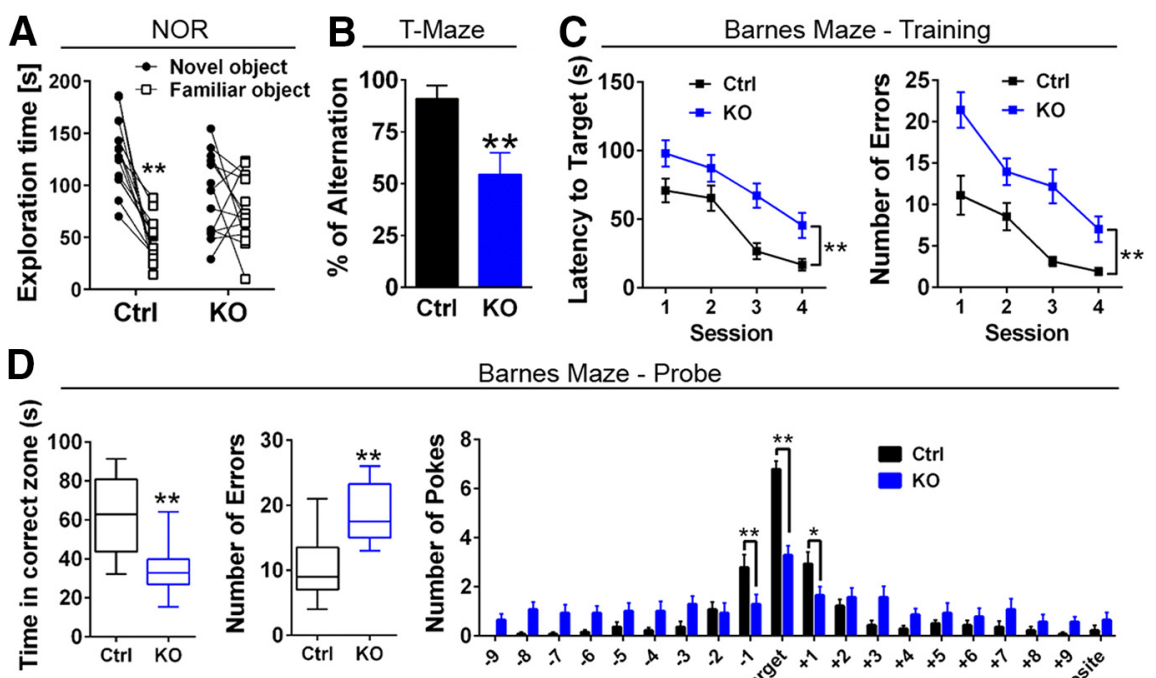

Barnes Maze - Probe
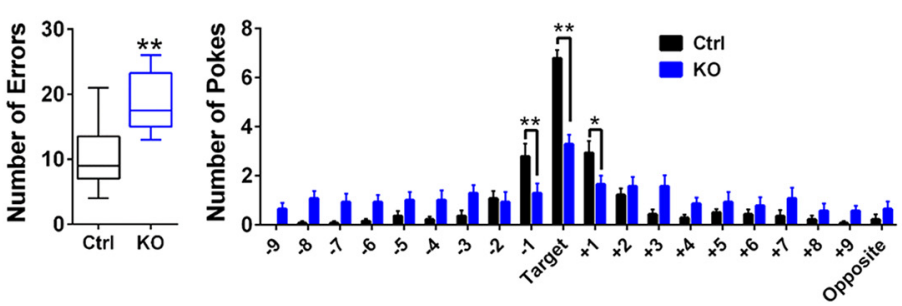

Figure 3. ErbB4 KO mice exhibit spatial learning and memory deficits. $\boldsymbol{A}$, ErbB4 KO mice $(n=13)$ show deficits during the NOR test as compared with control mice (Ctrl, $n=13$ ). ErbB4 KO mice were impaired to differentiate between the novel versus familiar object, assessed by the total time each object was explored during the probe session. $\boldsymbol{B}$, In contrast to control mice (Ctrl, black, $n=11$ ), ErbB4 KO mice (KO, blue, $n=11$ ) had lower spontaneous alternation in the T-maze. $\boldsymbol{C}$, Barnes maze $(n=14 / \mathrm{genotype})$ revealed learning deficits in ErbB4 $\mathrm{KO}$ mice to locate the target during the training sessions (days 1-4), including higher latency time to find the target (left panel) and more errors (right panel). $\boldsymbol{D}$, Consistently, the probe session (day 5) indicated that ErbB4 KO mice were impaired to locate the target, as the time spent in the correct zone was reduced (left panel), the number of incorrect nose pokes (errors) were higher (middle panel), and the number of nose pokes in the target and holes +1 and -1 were significantly lower than controls (right panel); $* p<0.05, * * p<0.01$.

\section{ErbB4 KO mice exhibit deficits in spatial learning memory}

Numerous studies in humans (Egan et al., 2001; Cools and D'Esposito, 2011), non-human primates (Arnsten et al., 1994; Williams and Goldman-Rakic, 1995), and rodents (Spellman et al., 2015; Cassidy et al., 2016) have shown the importance of an "inverted U-shape" relationship between optimal DA levels in PFC and performance on cognitive-related tasks. Based on this relationship, we reasoned that performance of ErbB4 KOs could be affected in hippocampal-dependent and PFC-dependent tasks. We therefore compared ErbB4 KOs and Ctrl in a battery of behavioral tasks to evaluate learning, spatial, and working memory reliant on these regions. ErbB4 KOs $(n=12)$ underscored relative to Ctrl $(n=12)$ in NOR (Fig. $3 A$ ), as ErbB4 KOs randomly explored both novel and familiar objects during the probe session (novel $91 \pm 11 \mathrm{~s}$ vs familiar $75 \pm 9 \mathrm{~s} ; p>0.05$ ), whereas Ctrl mice spent significantly more time with novel objects (novel $129 \pm 10 \mathrm{~s}$ vs familiar $\left.46 \pm 6 \mathrm{~s} ; t_{(12)}=6.47, p<0.0001\right)$. Additionally, ErbB4 KO mice failed to spontaneously alternate to explore both arms between consecutive trials in the T-maze $(54.6 \pm 10.3 \%, n=11)$, in contrast to Ctrl mice (Ctrl: $90.9 \pm 6.5 \%, n=11$ ), indicating deficits in working memory $(U=21.5, p=0.0055$; Fig. $3 B)$. Finally, assessing spatial learning using the Barnes maze, we found that ErbB4 KOs $(n=14)$ are impaired in learning the location of the target hole relative to Ctrl, as manifested by the increased latency times $\left(F_{(1,26)}=11.56, p=0.0022\right)$ and errors $\left(F_{(1,26)}=\right.$ 20.13, $p=0.0001$ ) per session as compared with Ctrl $(n=14$; Fig. $3 C)$. Moreover, ErbB4 KOs showed deficits in spatial memory during the Barnes maze probe session
(Fig. 3D), as suggested by the reduced time mice spent in the correct zone (ErbB4 KO $35 \pm 13 \mathrm{~s}$ vs Ctrl $63 \pm 5 \mathrm{~s} ; t_{(26)}=$ $\left.4.478, t_{(26)}=4.478, p<0.0001\right)$, the higher number of nose pokes errors (ErbB4 KO $19 \pm 1$ vs Ctrl $11 \pm 1$; $t_{(26)}=4.528$, $p<0.0001$ ), and their overall lower correct/incorrect hole nose-pokes score $\left(F_{(1,520)}=4.663, p=0.0313\right)$. These findings suggest that the NRG-ErbB4 signaling pathway plays a relevant role in memory consolidation.

\section{ErbB4 KO mice show increased willingness to work for palatable food rewards unrelated to energy requirement mechanisms}

The mesolimbic DA pathway, which projects from VTA to the NAc, is critical for the signal incentive salience regulating reinforcement/reward-related motor function learning (Schultz et al., 1997; Mohebi et al., 2019). Importantly, alterations in this pathway have been implicated in many human disorders including Parkinson's disease, schizophrenia and drug addiction (Salamone et al., 2007; Weinstein et al., 2017). Because we found NAc DA levels are reduced in ErbB4 KO mice (Fig. 1G), we assessed whether this altered pattern of DA was associated with behavioral changes in motivation and reinforcement learning. We initially tested anhedonia in mice using the two-bottle sucrose preference test (Nestler and Hyman, 2010). Consistent with prior observations (Shamir et al., 2012), we found that ErbB4 KOs did not show altered preference for a sucrose solution as the ratio of sucrosewater consumption was similar to that of Ctrl mice (KO: $0.83 \pm 0.02, n=11 ;$ vs Ctrl: $0.80 \pm 0.01, n=12 ; p=$ 0.0525). Next, we performed a CPP task (Fig. 4) to assess 


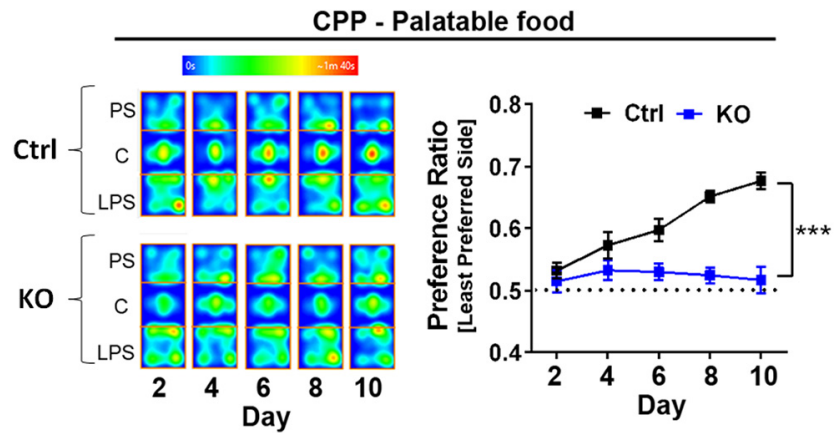

Figure 4. Cue-reward spatial association to palatable food is impaired in ErbB4 $\mathrm{KO}$ mice. To evaluate for spatial-cue associations to rewards, a five-test design (days 2, 4, 6, 8, and 10) was used to allow assessment of the development of a CPP to palatable food rewards. Food-restricted mice were conditioned to receive palatable food rewards (14-mg dustless pellets, BioServ) in a previously assigned LPS of the apparatus versus no food at the PS in two consecutive training sessions at days $1,3,5,7$, and 9 . The left panel is a representative heat map of ErbB4 KO (KO) and control (Ctrl) mice exploring the PS, center (C), and LPS of the apparatus across test sessions and the right panel shows the preference ratio of mice to develop CPP. Control mice $(n=15)$ progressively conditioned across sessions to spent more time in the LPS, yet ErbB4 KO mice $(n=16)$ did not develop spatial-cue associations to palatable food rewards (two-way ANVOA genotype effect: $F_{(1,29)}=32.78, p<0.0001$, two-way ANVOA time effect: $\left.F_{(4,116)}=8.619, p<0.0001\right)$. Data represents the mean $\pm \mathrm{SEM} ; * * * p<0.005$.

the development of reward-location associations. We found that, in stark contrast to the robust reward-associated place preference manifested by Ctrl mice $(n=15)$, ErbB4 KOs $(n=16)$ did not exhibit place preference association to palatable food pellets (two-way ANOVA genotype effect: $F_{(1,29)}=32.78, p<0.0001$, two-way ANOVA time effect: $F_{(4,116)}=8.619, p<0.0001$; Fig. 4). While these results could be interpreted as a failure of ErbB4 KOs to form cue-reward associations, previous reports have emphasized that CPP in rodents heavily relies on a preserved hippocampal system for spatial navigation (Ferbinteanu and McDonald, 2001; White et al., 2005; Ito et al., 2008). Because we observed that ErbB4 KOs exhibit deficits in spatial memory (Fig. $3 C, D$ ), we reasoned it was important to use other cue-reward behavioral paradigms before interpreting our CPP findings.

As alternatives to CPP, we used an auditory classical conditioning Pavlovian task and PR paradigm to model cue-reward associations, instrumental learning and reward in ErbB4 KOs (Salamone and Correa, 2002; Berridge and Robinson, 2003). The classical conditioning Pavlovian task did not uncover deficits in associative learning behaviors between genotypes (two-way ANOVA: $\left.F_{(1,13)}=1274, p=0.2794\right)$, as ErbB4 KOs $(n=7)$ and Ctrls $(n=8)$ learned similarly to associate an auditory cue-tone with the delivery of a palatable food reward across consecutive daily training sessions (two-way ANOVA: $F_{(7,91)}=$ $170.7, p<0.0001)$. Next, to evaluate instrumental learning, we trained mice to nose-poke a hole to earn a palatable food reinforcer under FR schedules. We found that ErbB4 KOs $(n=13)$ reached criteria faster than Ctrls $(n=12)$ at multiple FR schedules: FR1 (ErbB4 KO $3.4 \pm 0.1 \mathrm{~d}$ vs Ctrl $\left.5.2 \pm 0.2 \mathrm{~d}, t_{(23)}=7.22, p<0.0001\right)$, FR3 (ErbB4 KO $3.2 \pm 0.2 \mathrm{~d}$ vs Ctrl $3.9 \pm 0.3 \mathrm{~d}, t_{(23)}=2.242$, $p=0.0174$ ), and FR5 (ErbB4 KO $3.0 \pm 0.0 \mathrm{~d}$ vs Ctrl $\left.3.7 \pm 0.4 d, t_{(23)}=1.849, p=0.0387\right)$. Next, mice underwent a PR schedule to further evaluate their willingness to work for rewards (Fig. 5A). Unexpectedly, ErbB4 KOs outperformed $\mathrm{Ctrl}$ mice as defined by their higher break point average across five consecutive probe sessions $\left(F_{(1,23)}=\right.$ $5.565, p=0.0272$; Fig. 5B). Importantly, the performance of ErbB4 KO and Ctrl mice to retrieve rewards was goal directed, as the number of nose pokes to the active hole was significantly higher than to the inactive hole $\left(F_{(1,23)}=\right.$ $6.332, p=0.0193$; Fig. $5 C$ ). Taken together, these findings suggest that ErbB4 KOs do not show deficits in cue-reward associations and instrumental learning, as would be suggested by the CPP results alone (Fig. 4). Instead, ErbB4 KOs are more motivated to work for palatable food rewards than their Ctrl littermates (Fig. 5).

Given the apparent augmented willingness of ErbB4 KOs to work for palatable food under operant conditions, we considered whether these differences were primarily associated with intrinsic body metabolic requirements or, whether they were mediated by elevated motivation or appetite for palatable food. Accordingly, we initially monitored the body weight of ErbB4 KO and Ctrl mice $(n=7 /$ genotype) provided with regular chow and water ad libitum in their home cage every $30 \mathrm{~d}$ for a full year, and we found no differences between genotypes $(p>0.05$; Fig. $6 A)$. In separate cohorts of mice ( $n=13 /$ genotype), we then compared the energy balance (intake vs expenditure) and body mass composition (fat vs lean mass) of ErbB4 KOs and Ctrls for two consecutive weeks. Consistent with the previous observation (Fig. $6 A$ ), daily measurements of their body weight (ErbB4 KO $28.7 \pm 2.1 \mathrm{~g}$, Ctrl $26.8 \pm 1.4 \mathrm{~g}, p>0.05$; Fig. $6 B$ ), energy intake (ErbB4 KO $43.3 \pm 3.9 \mathrm{kcal} / \mathrm{BW}$, Ctrl $39.3 \pm 2.5 \mathrm{kcal} /$ BW, $p>0.05$; Fig. $6 C$ ) and expenditure (ErbB4 KO $44.9 \pm 3.2 \mathrm{kcal} / \mathrm{BW}$, Ctrl $41.8 \pm 2.1 \mathrm{kcal} / \mathrm{BW}, p>0.05$; Fig. $6 D$ ), and body mass composition (fat mass: ErbB4 KO $3.3 \pm 0.7 \mathrm{~g}$, Ctrl $3.3 \pm 0.5 \mathrm{~g}, p>0.05$; lean mass: ErbB4 KO $24.2 \pm 1.6 \mathrm{~g}$, Ctrl $23.1 \pm 1.1 \mathrm{~g}, p>0.05$; Fig. $6 E$ ) did not uncover differences between genotypes. To the extent assessed here, our data suggest that ErbB4 KOs and Ctrl mice do not show differences in their energy balance and body composition.

\section{Discussion}

Despite the emerging interest in NRG-ErbB signaling in DA function, how mutation of ErbB4 affects tonic DA levels in distinct brain areas and its association with behavioral alterations has remained mostly unknown. Here, we have demonstrated that ErbB4 KOs manifest an imbalance of steady-state extracellular DA across nigrostriatal and meso-cortico-limbic systems. Moreover, we confirm and expand on behavioral deficits observed in ErbB4 KOs relevant to psychiatric disorders, including increased locomotor activity, cognitive-related impairments, and elevated motivation/willingness to retrieve food rewards. 

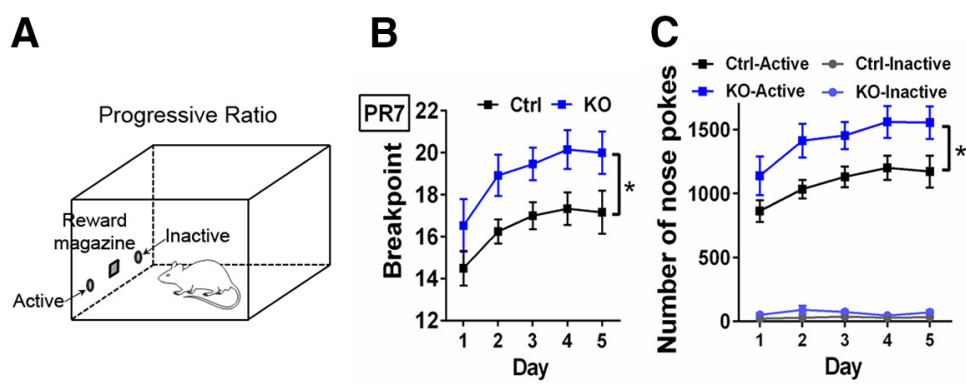

Figure 5. ErbB4 KO mice show increased motivation to work for palatable rewards. $\boldsymbol{A}$, Experimental set-up of a PR (PR7) schedule in food-restricted ( $\sim 85 \%$ free-food body weight) ErbB4 KO $(\mathrm{KO}, n=13)$ and control $(\mathrm{Ctrl}, n=12)$ mice. The PR7 is an instrumental learning paradigm where mice are trained to nose poke an active hole to retrieve a palatable reward (14-mg dustless precision pellet, BioServ) that increasingly becomes more difficult to obtain with each subsequent reward delivery (first pellet at seven nose pokes, second pellet at 14 nose pokes, etc.). B. Interestingly, ErbB4 KO mice show increased motivation to obtain palatable food rewards than Ctrl mice as their break point (number of rewards collected in a total of $120 \mathrm{~min}$ ) was consistently larger at each session (days $1-5 ; F_{(1,23)}=5.565, p=0.0272$ ). $C$, The larger number of rewards obtained by ErbB4 KO mice were attributed to goal-directed instrumental behaviors, and not to an overall increased locomotor activity because ErbB4 KO mice specifically and more frequently nose poked the active hole than Ctrl mice $\left(F_{(1,23)}=6.332, p=0.0193\right)$. Data are expressed as the mean $\pm \mathrm{SEM} ; * p<0.05$.

\section{ErbB4 deletion alters DA homeostasis across nigrostriatal and mesocorticolimbic systems}

In the present study we report that ErbB4 KO mice have elevated tonic extracellular DA levels in the dorsal
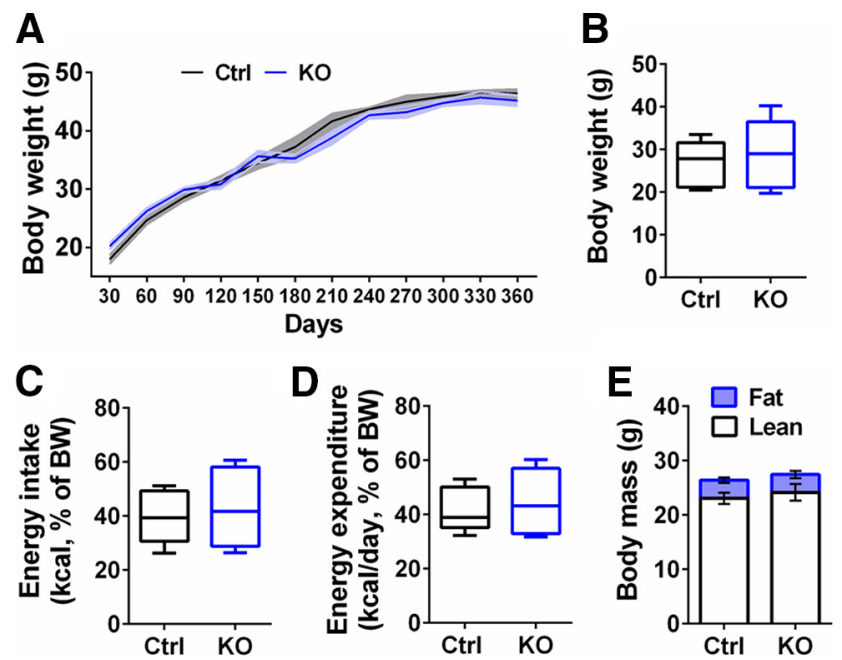

Figure 6. ErbB4 $\mathrm{KO}$ mice do not show differences in energy balance and body composition. $\boldsymbol{A}$, ErbB4 KO mice (KO, blue) kept with regular chow and water ad libitum in their home cages did not show differences with control (Ctrl, black) mice in body weight when continuously monitored every $30 \mathrm{~d}$ up to $360 \mathrm{~d}(n=7 /$ genotype, $p>0.05$ ). The shaded area around ErbB4 $\mathrm{KO}$ and Ctrl lines represent the SEM. $\boldsymbol{B}$, Moreover, an independent cohort of 90-d-old ErbB4 KO and Ctrl mice ( $n=13 / g e-$ notype) with free access to food and water were analyzed during 10 consecutive days for body weight, $(\boldsymbol{C})$ energy intake, $(\boldsymbol{D})$ energy expenditure, and $(\boldsymbol{E})$ body mass composition. In agreement to $\boldsymbol{A}, 90$-d-old ErbB4 $\mathrm{KO}$ and $\mathrm{Ctrl}$ mice have similar body weights $(\boldsymbol{p}>0.05 ; \boldsymbol{B})$. Consistently, the energy intake between ErbB4 KO and Ctrl mice $(\boldsymbol{p}>0.05 ; \boldsymbol{C})$ and energy expenditure did not show differences $(p>0.05 ; \boldsymbol{D})$. $\boldsymbol{E}$, Finally, no differences between fat/lean body mass composition was observed between genotypes $(p>0.05)$. Data are expressed as the mean \pm SEM. striatum, whereas they have reduced DA levels in mPFC, dorsal hippocampus, and NAc. These changes in tonic DA levels are similar to those observed in mice with targeted mutation of NRG2, a major ErbB ligand in the adult mouse brain (Yan et al., 2018). Interestingly, modulating ErbB receptor activity during neonatal development by systemically administering either NRG1 or a pan-ErbB inhibitor (JNJ-28871063) later cause alterations in DA levels in the adult (Kato et al., 2011; Mizuno et al., 2013; Golani et al., 2014). Moreover, it has recently been reported that targeted mutation of ErbB4 in tyrosine hydroxylase (TH) neurons, but not in PV + GABAergic interneurons, have decreased striatal and elevated mPFC-hippocampus tonic extracellular DA levels relative to controls (Skirzewski et al., 2018), a DA imbalance pattern that is inversed compared with the ErbB4 KOs described herein. Although we presently do not understand the mechanisms that account for the reciprocal changes of extracellular DA levels across brain regions observed in the pharmacological models, ErbB4 KO, NRG2 KO, and $\mathrm{TH}$-targeted ErbB4 $\mathrm{KO}$ mice, these findings suggest that alterations in NRG/ErbB4 signaling can bidirectionally affect the tonic levels of extracellular DA by affecting the activity of DAT in monoaminergic neurons (Skirzewski et al., 2018) and by altering the activity of local microcircuits. Recent retrograde and anterograde tracing studies, coupled to single-cell RNAseq analysis, have uncovered a previously unappreciated complexity of DA neurons that comprise nigrostriatal, mesocortical and mesolimbic connections (Morales and Root, 2014; Lammel et al., 2015; Menegas et al., 2015; Barker et al., 2016; Farassat et al., 2019). An interesting possibility that may account for the different effects of ErbB activity on tonic DA levels, is that NRG/ErbB4 signaling differs among distinct DA neuron populations. There is also evidence that mesocortico/limbic and nigrostriatal DA projections are mutually connected with cortical microcircuitry involving inhibitory GABAergic activity, particularly $\mathrm{PV}+$ fast-spiking interneurons (Uhlhaas and Singer, 2010; Lewis et al., 2011), that can regulate local microcircuits and levels of DA release. An interesting 
and important aspect of our findings of DA imbalance in ErbB4 and NRG2 KO mice, regardless of the underlying mechanisms, is that they are reminiscent of the DA imbalance reported in schizophrenia patients. In particular, our results recapitulate the hyperdopaminergic state in the striatum and the hypodopaminergic in the DLPFC often reported in schizophrenic patients (Weinstein et al., 2017). This is particularly critical, as it may suggest that the ineffectiveness of D2 receptortargeting antipsychotics in alleviating cognitive deficits could be associated with reduced neocortical dopaminergic function. Therefore, targeting NRG and ErbB4 mutations in mice to distinct neuronal subpopulations could provide an important model to uncover novel cellular targets and circuits that modulate DA levels in the neocortex, and that are necessary for optimal performance in cognitive tasks.

\section{ErbB4 is relevant to regulate cognitive function}

The findings presented here and earlier studies emphasize the importance of NRG/ErbB4 signaling for cognitive functions (Wen et al., 2010; Shamir et al., 2012; Loos et al., 2014, 2016; Marchisella et al., 2018; Skirzewski et al., 2018; Yan et al., 2018). Specifically, we found that ErbB4 $\mathrm{KO}$ mice show behavioral deficits that suggest impairments in declarative (NOR; Fig. $3 A$ ), spatial (Barnes maze; Fig. $3 C, D$ ), contextual (CPP; Fig. 4), and working memory functions (T-maze; Fig. 3B). Previous reports have shown that the medial temporal lobe system, which includes the hippocampus and the perirhinal, entorhinal, and parahippocampal cortices, is necessary to temporarily store factual information as declarative memory and is therefore relevant to encode locations and place-object associations (Squire et al., 2004). Moreover, these structures exhibit a widespread and reciprocal network connectivity with the neocortex to encode, maintain and retrieve relevant information necessary for spatial and working memory (Vann and Albasser, 2011; Spellman et al., 2015; Tamura et al., 2017). Lastly, cortical and hippocampal networks are regulated by GABAergic PV+ interneuron activity (Lewis et al., 2012; Gonzalez-Burgos et al., 2015; Dienel and Lewis, 2019) and are modulated by DA to regulate cognitive function (Williams and Goldman-Rakic, 1995; Goldman-Rakic et al., 2000; Winterer and Weinberger, 2004; Gonzalez-Burgos et al., 2005; Spellman et al., 2015; Cassidy et al., 2016).

Although we cannot establish a causal relationship between the cognitive deficits observed in ErbB4 KOs and the reductions in the relative levels of tonic extracellular DA in the mPFC and hippocampus (Fig. 1), it is tempting to speculate that these reduced levels of DA could contribute to the observed deficits in declarative, spatial, and working memory. It is well documented that DA plays an important role in modulating cognitive functions in primates (Arnsten et al., 2015), and DA receptor-targeting drugs are known to ameliorate acute motor and cognitive deficits in rodents with disruptions in the NRG-ErbB4 pathway (Sotoyama et al., 2011; Andersson et al., 2012; Yan et al., 2018). Reduced levels of DA in both MPFC and dorsal hippocampus of ErbB4 KOs could contribute to the observed deficits in declarative, spatial, and working memory. Interestingly, mice with targeted mutations of ErbB4 in $\mathrm{TH}+$ midbrain neurons manifest a relative increase of tonic extracellular DA levels in MPFC and dorsal hippocampus and also exhibit spatial and working memory deficits (Skirzewski et al., 2018). Of significance, targeted re-expression of ErbB4 in TH positive midbrain neurons of adult TH-ErbB4 $\mathrm{KO}$ mice resulted in a recovery of tonic extracellular DA levels and improvement in performance of spatial and working memory tasks (Skirzewski et al., 2018), consistent with an important association between optimal DA levels and cognitive performance.

However, one cannot rule out that other neurons also contribute to the association between ErbB4 and cognitive performance. Of particular relevance is the expression of ErbB4 in mPFC and hippocampal PV+ GABAergic interneurons, which express the highest relative levels of receptor and regulate $\gamma$ oscillation power (Vullhorst et al., 2009; Fazzari et al., 2010; Neddens et al., 2011). Mice with targeted mutation of ErbB4 in PV+ interneurons manifest working/spatial memory impairments (Wen et al., 2010; Del Pino et al., 2013), but they do not exhibit any changes in tonic extracellular DA levels in the neocortex or hippocampus (Skirzewski et al., 2018). Taken together, these results suggest that NRG-ErbB4 signaling in $\mathrm{TH}+$ neurons and $\mathrm{PV}+$ interneurons both contribute to sustain an optimal local network activity necessary for cognitive function in mice. Further analysis is warranted to better understand how the interaction between tonic extracellular DA levels and neuronal network activity, potentially mediated by cross-talk between DA D4 receptor and ErbB4 receptors on PV+ interneurons (Kwon et al., 2008; Andersson et al., 2012), regulate cognitive performance.

\section{ErbB4 regulates motivation for palatable food rewards}

The NAc is known as the limbic-motor interface in the central nervous system due to its role in integrating synaptic inputs from several brain regions to regulate goal-directed behaviors, motivation and reward (Mogenson et al., 1980; Groenewegen et al., 1999; Goto and Grace, 2008; Averbeck and Costa, 2017; Wise and McDevitt, 2018). Recent studies suggest that abnormal dopaminergic and GABAergic transmission in the NAc of rodents is associated with alterations in motivational functions (Stratford and Kelley, 1997; Reynolds and Berridge, 2001; Peciña et al., 2003; Rada et al., 2010; Smith et al., 2011; Beeler et al., 2012; Soares-Cunha et al., 2018), such as the exertion of effort during instrumental behavior, flexible approach behavior and exploitation of reward learning (Peciña et al., 2003; Rada et al., 2010; Smith et al., 2011; Beeler et al., 2012). As reported here, ErbB4 KO mice are more motivated than Ctrls to work for palatable food rewards by mechanisms unrelated to altered energy balance (Figs. 5, 6). Consistent with previous reports using systemic administration of JNJ28871063 (Golani et al., 2014; Tadmor et al., 2017, 2018), our findings show that NRG-ErbB4 signaling affects circuits that modulate reward-related behaviors. The regulation of motivational behaviors by NRG-ErbB4 signaling could potentially be attributed to changes in extracellular DA levels (this study) 
or to GABAergic transmission (Geng et al., 2017). A future challenge will be to identify the ErbB4-dependent mechanisms in the NAc and associated networks that drive this increased motivation to work for palatable food rewards.

\section{Concluding remarks}

This and prior reports have shown that the NRG-ErbB4 signaling modulates the dopaminergic and GABAergic neurotransmission systems. The functional interaction between these signaling pathways can contribute to regulate E/I balance, synaptic plasticity, synchrony network activity and phenotypes relevant to psychiatric disorders (Law et al., 2007; Buonanno, 2010; Kao et al., 2010; Greenwood et al., 2012; Joshi et al., 2014; Mei and Nave, 2014; Mostaid et al., 2016). Moreover, because the NRGErbB4 signaling pathway functionally interacts to regulate synaptic and network properties underlying complex behavioral traits, our findings highlight why classical therapeutic approaches targeting specific neurotransmitter pathways to treat the diverse symptoms in psychiatric disorders, including schizophrenia, have had relatively little success.

\section{References}

Andersson $\mathrm{RH}$, Johnston A, Herman PA, Winzer-Serhan UH, Karavanova I, Vullhorst D, Fisahn A, Buonanno A (2012) Neuregulin and dopamine modulation of hippocampal gamma oscillations is dependent on dopamine D4 receptors. Proc Natl Acad Sci USA 109:13118-13123.

Arnsten AF, Cai JX, Murphy BL, Goldman-Rakic PS (1994) Dopamine D1 receptor mechanisms in the cognitive performance of young adult and aged monkeys. Psychopharmacology (Berl) 116:143-151.

Arnsten AF, Wang M, Paspalas CD (2015) Dopamine's actions in primate prefrontal cortex: challenges for treating cognitive disorders. Pharmacol Rev 67:681-696.

Averbeck BB, Costa VD (2017) Motivational neural circuits underlying reinforcement learning. Nat Neurosci 20:505-512.

Bardo MT, Bowling SL, Pierce RC (1990) Changes in locomotion and dopamine neurotransmission following amphetamine, haloperidol, and exposure to novel environmental stimuli. Psychopharmacology (Berl) 101:338-343.

Barker DJ, Root DH, Zhang S, Morales M (2016) Multiplexed neurochemical signaling by neurons of the ventral tegmental area. $\mathrm{J}$ Chem Neuroanat 73:33-42.

Bean JC, Lin TW, Sathyamurthy A, Liu F, Yin DM, Xiong WC, Mei L (2014) Genetic labeling reveals novel cellular targets of schizophrenia susceptibility gene: distribution of GABA and non-GABA ErbB4-positive cells in adult mouse brain. J Neurosci 34:1354913566.

Beeler JA, Frazier CR, Zhuang X (2012) Dopaminergic enhancement of local food-seeking is under global homeostatic control. Eur $\mathrm{J}$ Neurosci 35:146-159.

Berridge KC, Robinson TE (2003) Parsing reward. Trends Neurosci 26:507-513.

Bertram I, Bernstein HG, Lendeckel U, Bukowska A, Dobrowolny H, Keilhoff G, Kanakis D, Mawrin C, Bielau H, Falkai P, Bogerts B (2007) Immunohistochemical evidence for impaired neuregulin-1 signaling in the prefrontal cortex in schizophrenia and in unipolar depression. Ann NY Acad Sci 1096:147-156.

Brennand KJ, Simone A, Jou J, Gelboin-Burkhart C, Tran N, Sangar S, Li Y, Mu Y, Chen G, Yu D, McCarthy S, Sebat J, Gage FH (2011) Modelling schizophrenia using human induced pluripotent stem cells. Nature 473:221-225.
Buonanno A (2010) The neuregulin signaling pathway and schizophrenia: from genes to synapses and neural circuits. Brain Res Bull 83:122-131.

Cassidy CM, Van Snellenberg JX, Benavides C, Slifstein M, Wang Z, Moore H, Abi-Dargham A, Horga G (2016) Dynamic connectivity between brain networks supports working memory: relationships to dopamine release and schizophrenia. J Neurosci 36:43774388.

Chang Q, Fischbach GD (2006) An acute effect of neuregulin 1 beta to suppress alpha 7-containing nicotinic acetylcholine receptors in hippocampal interneurons. J Neurosci 26:11295-11303.

Chen YJ, Zhang M, Yin DM, Wen L, Ting A, Wang P, Lu YS, Zhu XH, Li SJ, Wu CY, Wang XM, Lai C, Xiong WC, Mei L, Gao TM (2010) ErbB4 in parvalbumin-positive interneurons is critical for neuregulin 1 regulation of long-term potentiation. Proc Natl Acad Sci USA 107:21818-21823.

Chong VZ, Thompson M, Beltaifa S, Webster MJ, Law AJ, Weickert CS (2008) Elevated neuregulin-1 and ErbB4 protein in the prefrontal cortex of schizophrenic patients. Schizophr Res 100:270-280.

Cools R, D'Esposito M (2011) Inverted-U-shaped dopamine actions on human working memory and cognitive control. Biol Psychiatry 69:e113-e125.

Crawley J (2007) What's wrong with my mouse? In: Behavioral phenotyping of transgenic and knockout mice, Ed 2. Hoboken: WileyInterscience.

Deacon RM, Rawlins JN (2006) T-maze alternation in the rodent. Nat Protoc 1:7-12.

Del Pino I, García-Frigola C, Dehorter N, Brotons-Mas JR, AlvarezSalvado E, Martínez de Lagrán M, Ciceri G, Gabaldón MV, Moratal D, Dierssen M, Canals S, Marín O, Rico B (2013) Erbb4 deletion from fast-spiking interneurons causes schizophrenia-like phenotypes. Neuron 79:1152-1168.

Di Chiara G, Imperato A (1988) Drugs abused by humans preferentially increase synaptic dopamine concentrations in the mesolimbic system of freely moving rats. Proc Natl Acad Sci USA 85:52745278.

Dienel SJ, Lewis DA (2019) Alterations in cortical interneurons and cognitive function in schizophrenia. Neurobiol Dis 131:104208.

Egan MF, Goldberg TE, Kolachana BS, Callicott JH, Mazzanti CM, Straub RE, Goldman D, Weinberger DR (2001) Effect of COMT Val108/158 Met genotype on frontal lobe function and risk for schizophrenia. Proc Natl Acad Sci USA 98:6917-6922.

Farassat N, Costa KM, Stojanovic S, Albert S, Kovacheva L, Shin J, Egger R, Somayaji M, Duvarci S, Schneider G, Roeper J (2019) In vivo functional diversity of midbrain dopamine neurons within identified axonal projections. Elife 8:e48408.

Fazzari P, Paternain AV, Valiente M, Pla R, Luján R, Lloyd K, Lerma J, Marín O, Rico B (2010) Control of cortical GABA circuitry development by Nrg1 and ErbB4 signalling. Nature 464:1376-1380.

Ferbinteanu J, McDonald RJ (2001) Dorsal/ventral hippocampus, fornix, and conditioned place preference. Hippocampus 11:187200.

Fisahn A, Neddens J, Yan L, Buonanno A (2009) Neuregulin-1 modulates hippocampal gamma oscillations: implications for schizophrenia. Cereb Cortex 19:612-618.

Forwood SE, Winters BD, Bussey TJ (2005) Hippocampal lesions that abolish spatial maze performance spare object recognition memory at delays of up to 48 hours. Hippocampus 15:347-355.

Geng HY, Zhang J, Yang JM, Li Y, Wang N, Ye M, Chen XJ, Lian H, Li XM (2017) Erbb4 deletion from medium spiny neurons of the nucleus accumbens core induces schizophrenia-like behaviors via elevated GABAAR alpha1 expression. J Neurosci 37:7450-7464.

Golani I, Tadmor H, Buonanno A, Kremer I, Shamir A (2014) Disruption of the ErbB signaling in adolescence increases striatal dopamine levels and affects learning and hedonic-like behavior in the adult mouse. Eur Neuropsychopharmacol 24:1808-1818.

Goldman-Rakic PS, Muly EC 3rd, Williams GV (2000) D(1) receptors in prefrontal cells and circuits. Brain Res Brain Res Rev 31:295301. 
Gonzalez-Burgos G, Kroener S, Seamans JK, Lewis DA, Barrionuevo G (2005) Dopaminergic modulation of short-term synaptic plasticity in fast-spiking interneurons of primate dorsolateral prefrontal cortex. J Neurophysiol 94:4168-4177.

Gonzalez-Burgos G, Cho RY, Lewis DA (2015) Alterations in cortical network oscillations and parvalbumin neurons in schizophrenia. Biol Psychiatry 77:1031-1040.

Goto Y, Grace AA (2008) Limbic and cortical information processing in the nucleus accumbens. Trends Neurosci 31:552-558.

Greenwood TA, Light GA, Swerdlow NR, Radant AD, Braff DL (2012) Association analysis of 94 candidate genes and schizophrenia-related endophenotypes. PLoS One 7:e29630.

Grieco SF, Holmes TC, Xu X (2019) Neuregulin directed molecular mechanisms of visual cortical plasticity. J Comp Neurol 527:668678.

Groenewegen HJ, Wright Cl, Beijer AV, Voorn P (1999) Convergence and segregation of ventral striatal inputs and outputs. Ann NY Acad Sci 877:49-63.

Guo J, Hall KD (2009) Estimating the continuous-time dynamics of energy and fat metabolism in mice. PLoS Comput Biol 5: e1000511.

Hayes LN, Shevelkin A, Zeledon M, Steel G, Chen PL, Obie C, Pulver A, Avramopoulos D, Valle D, Sawa A, Pletnikov MV (2016) Neuregulin 3 knockout mice exhibit behaviors consistent with psychotic disorders. Mol Neuropsychiatry 2:79-87.

Hernandez L, Stanley BG, Hoebel BG (1986) A small, removable microdialysis probe. Life Sci 39:2629-2637.

Hutchison MA, Gu X, Adrover MF, Lee MR, Hnasko TS, Alvarez VA, Lu W (2018) Genetic inhibition of neurotransmission reveals role of glutamatergic input to dopamine neurons in high-effort behavior. Mol Psychiatry 23:1213-1225.

Ito R, Robbins TW, Pennartz CM, Everitt BJ (2008) Functional interaction between the hippocampus and nucleus accumbens shell is necessary for the acquisition of appetitive spatial context conditioning. J Neurosci 28:6950-6959.

Janssen MJ, Leiva-Salcedo E, Buonanno A (2012) Neuregulin directly decreases voltage-gated sodium current in hippocampal ErbB4-expressing interneurons. J Neurosci 32:13889-13895.

Joshi D, Fullerton JM, Weickert CS (2014) Elevated ErbB4 mRNA is related to interneuron deficit in prefrontal cortex in schizophrenia. J Psychiatr Res 53:125-132.

Kao WT, Wang Y, Kleinman JE, Lipska BK, Hyde TM, Weinberger DR, Law AJ (2010) Common genetic variation in Neuregulin 3 (NRG3) influences risk for schizophrenia and impacts NRG3 expression in human brain. Proc Natl Acad Sci USA 107:1561915624.

Kato T, Kasai A, Mizuno M, Fengyi L, Shintani N, Maeda S, Yokoyama M, Ozaki M, Nawa H (2010) Phenotypic characterization of transgenic mice overexpressing neuregulin-1. PLoS One 5: e14185.

Kato T, Abe Y, Sotoyama H, Kakita A, Kominami R, Hirokawa S, Ozaki M, Takahashi H, Nawa H (2011) Transient exposure of neonatal mice to neuregulin-1 results in hyperdopaminergic states in adulthood: implication in neurodevelopmental hypothesis for schizophrenia. Mol Psychiatry 16:307-320.

Kawata M, Morikawa S, Shiosaka S, Tamura H (2017) Ablation of neuropsin-neuregulin 1 signaling imbalances ErbB4 inhibitory networks and disrupts hippocampal gamma oscillation. Transl Psychiatry 7:e1052.

Kwon OB, Longart M, Vullhorst D, Hoffman DA, Buonanno A (2005) Neuregulin-1 reverses long-term potentiation at CA1 hippocampal synapses. J Neurosci 25:9378-9383.

Kwon OB, Paredes D, Gonzalez CM, Neddens J, Hernandez L, Vullhorst D, Buonanno A (2008) Neuregulin-1 regulates LTP at CA1 hippocampal synapses through activation of dopamine D4 receptors. Proc Natl Acad Sci USA 105:15587-15592.

Lammel S, Steinberg EE, Földy C, Wall NR, Beier K, Luo L, Malenka RC (2015) Diversity of transgenic mouse models for selective targeting of midbrain dopamine neurons. Neuron 85:429-438.
Law AJ, Lipska BK, Weickert CS, Hyde TM, Straub RE, Hashimoto R, Harrison PJ, Kleinman JE, Weinberger DR (2006) Neuregulin 1 transcripts are differentially expressed in schizophrenia and regulated by 5' SNPs associated with the disease. Proc Natl Acad Sci USA 103:6747-6752.

Law AJ, Kleinman JE, Weinberger DR, Weickert CS (2007) Diseaseassociated intronic variants in the ErbB4 gene are related to altered ErbB4 splice-variant expression in the brain in schizophrenia. Hum Mol Genet 16:129-141.

Lewis DA, Fish KN, Arion D, Gonzalez-Burgos G (2011) Perisomatic inhibition and cortical circuit dysfunction in schizophrenia. Curr Opin Neurobiol 21:866-872.

Lewis DA, Curley AA, Glausier JR, Volk DW (2012) Cortical parvalbumin interneurons and cognitive dysfunction in schizophrenia. Trends Neurosci 35:57-67.

Li KX, Lu YM, Xu ZH, Zhang J, Zhu JM, Zhang JM, Cao SX, Chen XJ, Chen Z, Luo JH, Duan S, Li XM (2011) Neuregulin 1 regulates excitability of fast-spiking neurons through Kv1.1 and acts in epilepsy. Nat Neurosci 15:267-273.

Loos M, Mueller T, Gouwenberg Y, Wijnands R, van der Loo RJ; Neuro-BSIK Mouse Phenomics Consortium, Birchmeier C, Smit AB, Spijker S (2014) Neuregulin-3 in the mouse medial prefrontal cortex regulates impulsive action. Biol Psychiatry 76:648-655.

Loos M, Schetters D, Hoogeland M, Spijker S, de Vries TJ, Pattij T (2016) Prefrontal cortical neuregulin-ErbB modulation of inhibitory control in rats. Eur J Pharmacol 781:157-163.

Lu Y, Sun XD, Hou FQ, Bi LL, Yin DM, Liu F, Chen YJ, Bean JC, Jiao HF, Liu X, Li BM, Xiong WC, Gao TM, Mei L (2014) Maintenance of GABAergic activity by neuregulin 1-ErbB4 in amygdala for fear memory. Neuron 84:835-846.

Marchisella E, Wijnands R, Koopmans B, Spijker S, Loos M (2018) Constitutive loss and acute pharmacological manipulation of ErbB4 signaling do not affect attention and inhibitory control in mice. Genes Brain Behav 17:56-69.

Mei L, Nave KA (2014) Neuregulin-ERBB signaling in the nervous system and neuropsychiatric diseases. Neuron 83:27-49.

Menegas W, Bergan JF, Ogawa SK, Isogai Y, Umadevi Venkataraju K, Osten P, Uchida N, Watabe-Uchida M (2015) Dopamine neurons projecting to the posterior striatum form an anatomically distinct subclass. Elife 4:e10032.

Miller EK, Lundqvist M, Bastos AM (2018) Working memory 2.0. Neuron 100:463-475.

Mitchell RM, Janssen MJ, Karavanova I, Vullhorst D, Furth K, Makusky A, Markey SP, Buonanno A (2013) ErbB4 reduces synaptic GABAA currents independent of its receptor tyrosine kinase activity. Proc Natl Acad Sci USA 110:19603-19608.

Mizuno M, Sotoyama $H$, Namba $H$, Shibuya M, Eda T, Wang R, Okubo T, Nagata K, Iwakura Y, Nawa H (2013) ErbB inhibitors ameliorate behavioral impairments of an animal model for schizophrenia: implication of their dopamine-modulatory actions. Transl Psychiatry 3:e252.

Mogenson GJ, Jones DL, Yim CY (1980) From motivation to action: functional interface between the limbic system and the motor system. Prog Neurobiol 14:69-97.

Mohebi A, Pettibone JR, Hamid AA, Wong JT, Vinson LT, Patriarchi T, Tian L, Kennedy RT, Berke JD (2019) Dissociable dopamine dynamics for learning and motivation. Nature 570:65-70.

Morales M, Root DH (2014) Glutamate neurons within the midbrain dopamine regions. Neuroscience 282:60-68.

Mostaid MS, Lloyd D, Liberg B, Sundram S, Pereira A, Pantelis C, Karl T, Weickert CS, Everall IP, Bousman CA (2016) Neuregulin-1 and schizophrenia in the genome-wide association study era. Neurosci Biobehav Rev 68:387-409.

Namba H, Okubo T, Nawa H (2016) Perinatal exposure to neuregulin-1 results in disinhibition of adult midbrain dopaminergic neurons: implication in schizophrenia modeling. Sci Rep 6:22606.

Neddens J, Buonanno A (2010) Selective populations of hippocampal interneurons express ErbB4 and their number and distribution is altered in ErbB4 knockout mice. Hippocampus 20:724-744. 
Neddens J, Fish KN, Tricoire L, Vullhorst D, Shamir A, Chung W, Lewis DA, McBain CJ, Buonanno A (2011) Conserved interneuronspecific ErbB4 expression in frontal cortex of rodents, monkeys, and humans: implications for schizophrenia. Biol Psychiatry 70:636-645.

Nestler EJ, Hyman SE (2010) Animal models of neuropsychiatric disorders. Nat Neurosci 13:1161-1169.

Paxinos G, Franklin K (2001) The mouse brain in stereotaxic coordinates, Ed 2. San Diego: Elsevier Academic Press.

Peciña S, Cagniard B, Berridge KC, Aldridge JW, Zhuang X (2003) Hyperdopaminergic mutant mice have higher "wanting" but not "liking" for sweet rewards. J Neurosci 23:9395-9402.

Rada P, Bocarsly ME, Barson JR, Hoebel BG, Leibowitz SF (2010) Reduced accumbens dopamine in Sprague-Dawley rats prone to overeating a fat-rich diet. Physiol Behav 101:394-400.

Ravussin Y, Gutman R, LeDuc CA, Leibel RL (2013) Estimating energy expenditure in mice using an energy balance technique. Int $\mathrm{J}$ Obes (Lond) 37:399-403.

Reynolds SM, Berridge KC (2001) Fear and feeding in the nucleus accumbens shell: rostrocaudal segregation of GABA-elicited defensive behavior versus eating behavior. J Neurosci 21:32613270 .

Salamone JD, Correa M (2002) Motivational views of reinforcement: implications for understanding the behavioral functions of nucleus accumbens dopamine. Behav Brain Res 137:3-25.

Salamone JD, Correa M, Farrar A, Mingote SM (2007) Effort-related functions of nucleus accumbens dopamine and associated forebrain circuits. Psychopharmacology (Berl) 191:461-482.

Schultz W, Dayan P, Montague PR (1997) A neural substrate of prediction and reward. Science 275:1593-1599.

Segal DS (1975) Behavioral characterization of d- and I-amphetamine: neurochemical implications. Science 190:475-477.

Shamir A, Kwon OB, Karavanova I, Vullhorst D, Leiva-Salcedo E, Janssen MJ, Buonanno A (2012) The importance of the NRG-1/ ErbB4 pathway for synaptic plasticity and behaviors associated with psychiatric disorders. J Neurosci 32:2988-2997.

Skirzewski M, Karavanova I, Shamir A, Erben L, Garcia-Olivares J, Shin JH, Vullhorst D, Alvarez VA, Amara SG, Buonanno A (2018) ErbB4 signaling in dopaminergic axonal projections increases extracellular dopamine levels and regulates spatial/working memory behaviors. Mol Psychiatry 23:2227-2237.

Smith KS, Berridge KC, Aldridge JW (2011) Disentangling pleasure from incentive salience and learning signals in brain reward circuitry. Proc Natl Acad Sci USA 108:E255-E264.

Soares-Cunha C, Coimbra B, Domingues AV, Vasconcelos N, Sousa $\mathrm{N}$, Rodrigues AJ (2018) Nucleus accumbens microcircuit underlying D2-MSN-driven increase in motivation. eNeuro 5:ENEURO.038618.2018.

Sotoyama $H$, Zheng $Y$, Iwakura $Y$, Mizuno M, Aizawa $M$, Shcherbakova K, Wang R, Namba H, Nawa H (2011) Pallidal hyperdopaminergic innervation underlying D2 receptor-dependent behavioral deficits in the schizophrenia animal model established by EGF. PLoS One 6:e25831.

Spellman T, Rigotti M, Ahmari SE, Fusi S, Gogos JA, Gordon JA (2015) Hippocampal-prefrontal input supports spatial encoding in working memory. Nature 522:309-314.

Squire LR, Stark CE, Clark RE (2004) The medial temporal lobe. Annu Rev Neurosci 27:279-306.

Stratford TR, Kelley AE (1997) GABA in the nucleus accumbens shell participates in the central regulation of feeding behavior. $J$ Neurosci 17:4434-4440.

Sun Y, Ikrar T, Davis MF, Gong N, Zheng X, Luo ZD, Lai C, Mei L, Holmes TC, Gandhi SP, Xu X (2016) Neuregulin-1/ErbB4 signaling regulates visual cortical plasticity. Neuron 92:160-173.
Sunyer B, Patil S, Höger H, Lubec G (2007) Barnes maze, a useful task to assess spatial reference memory in the mice. Protocol Exchange. doi:10.1038/nprot.2007.390.

Tadmor H, Golani I, Dvir E, Kremer I, Shamir A (2017) Behavioral characterization of blocking the ErbB signaling during adolescent and adulthood in reward-liking (preference) and reward-related learning. Behav Brain Res 326:139-146.

Tadmor H, Golani I, Doron R, Kremer I, Shamir A (2018) ErbB signaling antagonist ameliorates behavioral deficit induced by phencyclidine (PCP) in mice, without affecting metabolic syndrome markers. Prog Neuropsychopharmacol Biol Psychiatry 82:322331.

Tamura M, Spellman TJ, Rosen AM, Gogos JA, Gordon JA (2017) Hippocampal-prefrontal theta-gamma coupling during performance of a spatial working memory task. Nat Commun 8:2182.

Tan Z, Robinson HL, Yin DM, Liu Y, Liu F, Wang H, Lin TW, Xing G, Gan L, Xiong WC, Mei L (2018) Dynamic ErbB4 activity in hippocampal-prefrontal synchrony and top-down attention in rodents. Neuron 98:380-393.e4.

Tidcombe H, Jackson-Fisher A, Mathers K, Stern DF, Gassmann M, Golding JP (2003) Neural and mammary gland defects in ErbB4 knockout mice genetically rescued from embryonic lethality. Proc Natl Acad Sci USA 100:8281-8286.

Uhlhaas PJ, Singer W (2010) Abnormal neural oscillations and synchrony in schizophrenia. Nat Rev Neurosci 11:100-113.

Vann SD, Albasser MM (2011) Hippocampus and neocortex: recognition and spatial memory. Curr Opin Neurobiol 21:440-445.

Vullhorst D, Neddens J, Karavanova I, Tricoire L, Petralia RS, McBain CJ, Buonanno A (2009) Selective expression of ErbB4 in interneurons, but not pyramidal cells, of the rodent hippocampus. J Neurosci 29:12255-12264.

Vullhorst D, Mitchell RM, Keating C, Roychowdhury S, Karavanova I, Tao-Cheng JH, Buonanno A (2015) A negative feedback loop controls NMDA receptor function in cortical interneurons via neuregulin 2/ErbB4 signalling. Nat Commun 6:7222.

Weinstein JJ, Chohan MO, Slifstein M, Kegeles LS, Moore H, AbiDargham A (2017) Pathway-specific dopamine abnormalities in schizophrenia. Biol Psychiatry 81:31-42.

Wen L, Lu YS, Zhu XH, Li XM, Woo RS, Chen YJ, Yin DM, Lai C, Terry AV Jr, Vazdarjanova A, Xiong WC, Mei L (2010) Neuregulin 1 regulates pyramidal neuron activity via ErbB4 in parvalbumin-positive interneurons. Proc Natl Acad Sci USA 107:1211-1216.

White NM, Chai SC, Hamdani S (2005) Learning the morphine conditioned cue preference: cue configuration determines effects of lesions. Pharmacol Biochem Behav 81:786-796.

Williams GV, Goldman-Rakic PS (1995) Modulation of memory fields by dopamine D1 receptors in prefrontal cortex. Nature 376:572575.

Winterer G, Weinberger DR (2004) Genes, dopamine and cortical signal-to-noise ratio in schizophrenia. Trends Neurosci 27:683-690.

Wise RA, McDevitt RA (2018) Drive and reinforcement circuitry in the brain: origins, neurotransmitters, and projection fields. Neuropsychopharmacology 43:680-689.

Yan L, Shamir A, Skirzewski M, Leiva-Salcedo E, Kwon OB, Karavanova I, Paredes D, Malkesman O, Bailey KR, Vullhorst D, Crawley JN, Buonanno A (2018) Neuregulin-2 ablation results in dopamine dysregulation and severe behavioral phenotypes relevant to psychiatric disorders. Mol Psychiatry 23:1233-1243.

Zhong C, Du C, Hancock M, Mertz M, Talmage DA, Role LW (2008) Presynaptic type III neuregulin 1 is required for sustained enhancement of hippocampal transmission by nicotine and for axonal targeting of alpha7 nicotinic acetylcholine receptors. J Neurosci 28:9111-9116. 